\title{
Comparison of Prone Hip Extension Exercise and Prone Hip Extension Exercise after Iliopsoas Stretching on Lumbopelvic Control and Gluteus Maximus Activity in Subjects with Short Iliopsoas
}

\author{
In-cheol Jeon, Ph.D., PT, Professor ${ }^{1}$; Jun-hyeok Jang, Ph.D., Professor ${ }^{2}$ \\ ${ }^{1}$ Department of Physical Therapy, Hoseo University, Asan, South Korea \\ ${ }^{2}$ Hill \& Heal Balance Center, Busan, South Korea
}

\begin{abstract}
Background Iliopsoas-stretching is frequently used to improve lumbopelvic control and hip movements in the clinical setting. However, little research into the effects of iliopsoas stretching in subjects with limited hip extension during prone hip extension exercise (PHE) has been reported on gluteus maximus activity and lumbopelvic motions.
\end{abstract}

Purpose To compare PHE and prone hip extension after iliopsoas stretching (PHEIS) on the electromyographic activity of gluteus maximus (GM) and angle of anterior tilt and rotation of lumbopelvic region and hip extension in subjects with a short iliopsoas.

Study design Pre-post intervention comparison.

Methods Sixteen-healthy males with a short iliopsoas participated in this study. Electromyography (EMG) was employed to investigate the GM, erector spinae (ES), multifidus (MF) and biceps femoris $(\mathrm{BF})$. The lumbopelvic compensations were monitored by an electromagnetic tracking motion analysis device. In addition, the hip extension angle was measured by the modified Thomas test. The significant difference was compared using paired $t$-test.

Results GM muscle activity and hip extension angle were significantly greater during PHEIS compared to PHE $(p<0.05)$. ES, BF and lumbopelvic compensations were significantly lower during PHEIS compared to PHE $(p<0.05)$.

Conclusions PHEIS can be an effective intervention of selectively strengthening the GM muscles with minimizing over-activity of ES and BF muscle and lumbopelvic compensations in subjects with a short iliopsoas.

Key words Gluteus maximus; Hip joint; Iliopsoas stretching; Lumbopelvic control; Prone hip extension.
Journal of KEMA 2017; 1(1): 19-25 Published Online Dec 30, 2017 pISSN: 2586-4351

Article History Received 02 November 2017 Revised 16 November 2017 (1st)

Revised 23 November 2017 (2nd)

Accepted 23 November 2017

\section{CONTACT}

jangpt@hanmail.net Jun-hyeok Jang, Hill \& Heal Balance Center, Heaundea-gu 433 beon gil 30-1 302, Busan, Republic of Korea

This is an Open-Access article distributed under the terms of the Creative Commons Attribution Non-Commercial License (http://creativecommons. org/licenses/by-nc/4.0) which permits unrestricted non-commercial use, distribution, and reproduction in any medium, provided the

\section{INTRODUCTION}

Iliopsoas muscle stretching is one of the primary important methods in hip joint rehabilitation. ${ }^{1}$ Shortness of the iliopsoas muscle was a main cause of faulty hip movement during prone hip extension (PHE). ${ }^{1}$ Reduced iliopsoas flexibility causes insufficient hip extension angle during PHE by anteriorly pulling on the lesser trochanter. ${ }^{2}$ As a result, limited hip extension range of motion (ROM) contributed to a possible cause of both increased anterior pelvic tilt and subsequent excessive lumbar lordosis during PHE. ${ }^{1}$ Therefore, the hyperextension of the lumbar spine with dominant back extensor 
muscle may result in facet joint impingement with the onset of low back pain (LBP). ${ }^{3}$

The main muscle involved in increasing hip extension angle is gluteus maximus (GM) in prone position. The GM plays an important role to maintain the axis of femur head with constant center of motion and to transfer the load through the pelvis during PHE. ${ }^{1}$ Incorrect PHE exercises because of shortness of the iliopsoas muscle may lead to increased adjacent muscle activation such as the biceps femoris (BF) and erector spinae (ES) and to uncontrolled lumbopelvic motions. Hyperactivity of the BF with ES resulting in insufficient GM muscle activity may be related to decreased hip extension angle in subjects with shortness of the iliopsoas. ${ }^{4}$ Thus, increasing the flexibility of iliopsoas with selective GM activation might be important for the correct PHE exercises in hip rehabilitation.

Many previous studies have investigated the effectiveness of various stretching exercises for increasing flexibility of iliopsoas. ${ }^{5,6}$ These studies have demonstrated immediately increased ROM of hip extension after stretching exercise. ${ }^{5,6}$ The previous study reported improving hip extension ROM may reduce this compensatory anterior pelvic tilt, thereby more evenly contributing to the performance demands on the lumbar spine. ${ }^{7}$ However, in this study, we used the modified stretching technique to control the amount of applied pressure to the hip joint with external lumbopelvic fixation by hand. This study used gross stretching in prone to increase the flexibility of iliopsoas.

Various interventions, including the conventional PHE, the modified PHE, the single-limb squat and the double-legged squat have been suggested to increase GM activation in individuals with GM weakness. ${ }^{8-10}$ In previous study, performing the PHE exercise with constant knee flexion of $90^{\circ}$ compared to constant full knee extension could be a better strategy to selectively activate GM muscle with minimal activation of BF and ST. ${ }^{11}$ In addition, Kang et al (2013) reported that PHE with knee flexion exercise in the $30^{\circ}$ hip abduction can lead to maximal GM and minimal hamstring activity than $0^{\circ}$ hip abduction position. For these reasons, PHE exercise was performed with $30^{\circ}$ hip abduction with $90^{\circ}$ knee flexion to increase the activity of GM. ${ }^{9,11}$

Iliopsoas stretching might be used to reduce muscle imbalance before exercises to activate GM muscle activity for subjects with a short iliopsoas. Even if this technique might be helpful, no studies have investigated to increase the flexibility of iliopsoas and the activation of GM muscle in subjects with a short iliopsoas. The purpose of present study was to compare the difference between PHE and prone hip extension exercise after iliopsoas stretching (PHEIS) on the electromyographic (EMG) activity of GM and angle of anterior tilt and rotation of pelvis and hip extension in subjects with a short iliopsoas. The hypothesis of this study was that GM activity and hip extension angle would be more increased and that ES, $\mathrm{BF}$ and lumbopelvic compensations would be more decreased during PHEIS compared to only PHE in subjects with a short iliopsoas.

\section{METHODS}

\section{Participants}

G-power analysis software was used with G*power software ver. 3.1.2 (Franz Faul, University of Kiel, Kiel, Germany) through the pilot study including ten subjects. The sample size was calculated with 0.80 power, 0.05 alpha level, and 1.48 effect size. This result indicated that a necessary sample size was at least 8 subjects for this study. Sixteen healthy male subjects were recruited in this study. ${ }^{12}$ All subjects had iliopsoas shortness as confirmed by the modified Thomas test. Their mean age was $21.4 \pm 1.2($ mean $\pm \mathrm{SD})$ years, their mean body weight was $63.1 \pm 5.1$, and their mean height was $171.6 \pm 1.8$. The exclusion criteria were as follows: 1 ) rectus femoris tightness as evidenced by the modified Thomas test ${ }^{13}$; 2) the anterior gliding syndrome of femur head was occurred during $\mathrm{PHE}^{1}$; 3 ) pain in any region of the body during testing. The protocols were explained in detail to the subjects, and provided an informed written consent and obtained from all subjects.

\section{Procedures}

The subjects were familiarized with the PHE with $30^{\circ}$ hip abduction with $90^{\circ}$ knee flexion during a 5-min period prior to measurements. The subjects were asked to be in prone on the table. Each subject was performed each task until hip extension angle $\left(10^{\circ}\right)$ was reached to the placement of the target bar. During all the exercises, the target bar was placed at 5 $\mathrm{cm}$ above the center of popliteal fossa. For the EMG data measurement, the subjects were asked to perform two trials of each exercise (PHE first and then PHEIS) with a $1 \mathrm{~min}$ resting time between trials. EMG data for $5 \mathrm{sec}$ were collected during the isometric phase of exercise. To analyze EMG data, middle $3 \mathrm{sec}$ during the isometric phase of each exercise was used to prevent possible starting or ending effects and connecting element of skin-electrode. ${ }^{14}$ The value of each trial was averaged for the data analysis. The metronome was used at one beat per second to perform exercises at standard speed. ${ }^{15}$ The subjects had resting time to avoid test order effect for 10 min after PHE. ${ }^{16}$ The hip extension angle was measured by the modified Thomas test after each PHE exercise. The examiners were blinded. The iliopsoas 
muscle stretching was supervised by a physical therapist with 7 years experience. The stretching was repeated 10 times. Each stretching was maintained for $30 \mathrm{sec}$. The resting time for $15 \mathrm{sec}$ was provided between each stretching. After the iliopsoas stretching, the same procedure was performed to measure EMG activities and hip extension angle.

\section{Electromyography recording and data analysis}

EMG-feedback was provided by a wireless telemetry system (Wireless EMG System (100RT), BTS, Millan, Italy). To analyze hip and trunk muscle contraction, we used surface EMG with the analyzing software. A digital band-pass filter (Lancosh FIR), which filtered movement artifacts were eliminated by a $20-450 \mathrm{~Hz}$. And sample rate was set 1,024 Hz. The EMG signals were processed with root mean square with a moving window of $50 \mathrm{~ms}$. While subjects maintained the dominant leg at the target bar, EMG signals were recorded for $5 \mathrm{sec}$. Before electrode placement, the skin was cleaned and swabbed by cotton with isopropyl alcohol to minimize skin resistance. Disposable $\mathrm{Ag} / \mathrm{AgCl}$ surface electrodes were applied to the exact sites. ${ }^{17,18}$ Electrodes were placed over the midsection of subjects' muscle bellies. ${ }^{14}$ Two electrodes were placed parallel to the target muscle fiber each on right ES, bilateral MF, right GM, right BF. ${ }^{11}$ For normalization of each muscle contraction, the manual muscle testing positions for MVIC of ES, MF, GM, and BF were performed. ${ }^{19}$

\section{Kinematics measurements}

The Polhemus Liberty ${ }^{\mathrm{TM}}$ (Polhemus, Colchester, VT, USA) was employed to investigate pelvic compensations at $120 \mathrm{~Hz}$ and monitor compensations of anterior tilting and rotation during the exercises. Metal objects were eliminated for interference effect. The electromagnetic motion sensor was attached to S2 skin. The transmitter was remained with the same position and orientation during all PHE exercises performed. The electromagnetic tracker system (global reference frame) was aligned with the orientation of PTHE, $+\mathrm{X}$ parallel to the line of both anterior superior iliac crest (ASIS), $+Y$ directed anterior-posterior axis, and $+Z$ vertically upwards.

\section{Hip extension angle measurement}

The subjects were positioned for the modified Thomas test in supine position at the edge of the therapeutic table. Hip extension angle was measured by universal goniometer. The axis of the goniometer was placed at greater trochanter in tested side. The linear bar was placed to femur. The ICC was 0.87 .

Prone hip extension
The subject performed a prone position on the table with his upper trunk, pelvis, and lower extremity in a straight line. Their feet were placed with shoulder apart. Both arms were placed comfortably beside the trunk without pushing the ground with the hands. The subject was asked to perform PHE in the prone position with $30^{\circ}$ hip abduction with $90^{\circ}$ knee flexion until his leg touched the bar (Figure 1). Thus, the hip joint was in extension at $10^{\circ}$ with knee flexion reached the target bar, maintained in the same position for 5 $\mathrm{s}$, and then slowly returned to the start position.

\section{Prone hip extension after iliopsoas stretching}

The subjects were positioned in a prone position, with their feet width shoulder apart and $90^{\circ}$ knee flexion of tested side. Their arms were comfortably placed beside trunk. The investigator stabilized the sacrum and tested side of ilium by placing one hand. And then, another hand grasped the $90^{\circ}$ bent knee and passively, vertically extended the subject's hip joint until the end range of motion for $30 \mathrm{sec}, 10$ times with $15 \mathrm{sec}$ resting time between each stretch (Figure 2).

\section{Statistical analysis}
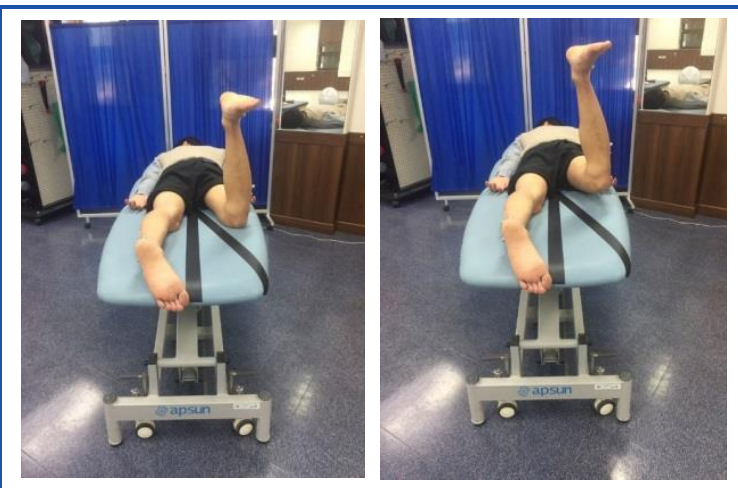

Figure 1. Prone hip extension.

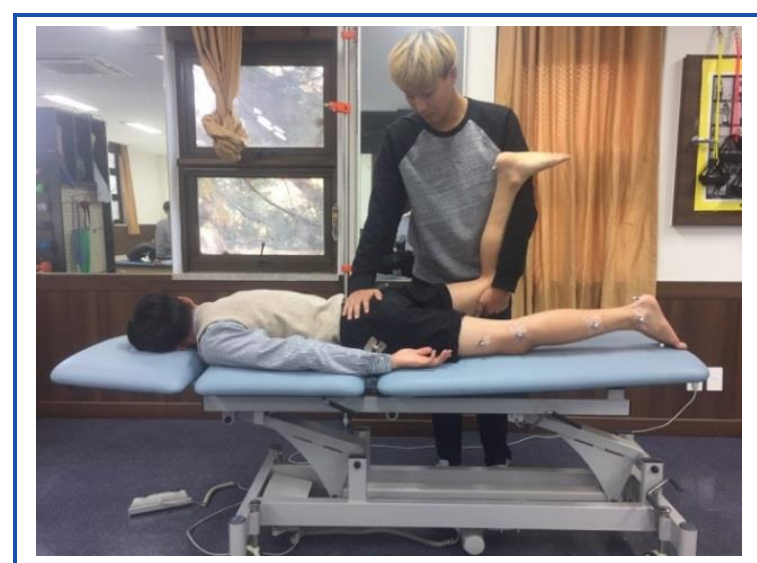

Figure 2. Iliopsoas stretching. 
To assess the normality of the distribution, a KolmogorovSmirnov test was employed. Differences in EMG muscle activities and lumbopelvic motions between each exercise (PHE and PHEIS) were compared using Paired $t$-tests. The level of significance was set at $\alpha=0.05$.

\section{RESULTS}

\section{EMG activity}

The ES EMG activities in both sides and BF EMG activities in right side were significantly lower in PHEIS than PHE $(p<0.05)$ (Table 1). The GM EMG activities in right side was significantly greater for PHEIS than PHE $(p<0.05)$ (Table 1). However, There was no significant difference in the EMG amplitudes in the both MF between the exercises $(p>0.05)$.

\section{Lumbopelvic kinematics}

The anterior tilting and rotation during PHEIS was significantly lower than PHE $(p<0.05)$ (Table 2).

\section{Hip extension}

The hip extension angle assessed by the modified Thomas test after iliopsoas stretching was significantly greater compared to PHE $(p<0.05)$ (Table 3$)$.

\section{DISCCUSSION}

The purpose of this study was to investigate the iliopsoas stretching in subjects with iliopsoas shortness by comparing
Table 3. Hip extension angle

\begin{tabular}{|c|c|c|c|c|}
\hline \multirow{2}{*}{$\begin{array}{l}\text { Hip joint } \\
\text { kinematics }\end{array}$} & \multicolumn{2}{|c|}{ Mean $\left(^{\circ}\right)$} & \multirow{2}{*}{$t$ value } & \multirow{2}{*}{$\begin{array}{c}p \\
\text { value }\end{array}$} \\
\hline & Pre & Post & & \\
\hline $\begin{array}{c}\text { Extension } \\
\left(^{\circ}\right)\end{array}$ & 3.6 & 9.8 & 5.74 & $<0.05$ \\
\hline
\end{tabular}

both PHE and PHEIS. This study compared EMG activities of ES, MF, GM and BF and lumbopelvic compensations. This study can be novel to investigate the hip extension angle and GM activity after stretching of iliopsoas during PHE.

Our results reported that the hip extension angle and GM activity were significantly greater and the lumbopelvic compensations, ES and BF were significantly lower in PHEIS compared with PHE. The changing differences in the hip extension angle, GM, ES and BF activity were $6.2^{\circ}, 28.31 \%$, $-13.87 \%$ and $-10.81 \%$ respectively. The previous study reported that the hold and relax technique of the iliopsoas muscle stretching decreased the back pain and lumbar lordosis angle, increased transvers abdominalis and hip extension angle in subjects with lumbar hyperlordosis. ${ }^{20}$ The different iliopsoas stretching techniques with both muscle energy technique and post isometric relaxation for 3 weeks in healthy subjects were investigated to compare the effectiveness on hip extension angle. ${ }^{21}$ The findings from the previous study reported that although both muscle energy technique and post isometric relaxation were effective for improving iliopsoas length, muscle energy technique was more effective compared to post isometric relaxation in hip extension angle assessed by the modified thomas test (Hip extension angle

Table 1. EMG activities of the various muscles

\begin{tabular}{ccccc}
\hline \multirow{2}{*}{ Muscles } & \multicolumn{2}{c}{ Mean $\pm^{\mathrm{i} S D}\left(\%{ }^{\mathrm{j} M V I C}\right)$} & \multirow{2}{*}{$t$ value } & \multirow{2}{*}{$p$ value } \\
\cline { 2 - 3 } & PHE & PHEIS & -7.82 & $0.03^{*}$ \\
\hline ES (Rt) & $24.08 \pm 9.84$ & $10.21 \pm 5.25$ & -4.52 & $0.02^{*}$ \\
ES (Lt) & $25.01 \pm 13.54$ & $17.34 \pm 10.72$ & 1.25 & 0.24 \\
MF (Rt) & $27.71 \pm 12.27$ & $29.94 \pm 15.99$ & -3.69 & 0.14 \\
MF (Lt) & $24.57 \pm 10.41$ & $20.74 \pm 8.10$ & 16.61 & $0.01^{*}$ \\
GM (Rt) & $28.02 \pm 11.75$ & $56.33 \pm 6.62$ & -5.20 & $0.02^{*}$ \\
\hline BF (Rt) & $30.12 \pm 25.14$ & $19.31 \pm 10.01$ &
\end{tabular}

PHE: prone hip extension, PHEIS: prone hip extension after iliopsoas stretching, ES (Rt): erector spinae in Rt. Side, ES (Lt): erector spinae in Lt. side, MF (Rt): multifidus in Rt. Side, MF (Lt): multifidus in Lt. Side, GM (Rt): gluteus maximus in Rt. Side, $\mathrm{BF}(\mathrm{Rt})$ : biceps femoris in Rt. Side, SD: standard deviation, MVIC: maximal voluntary isometric contraction.

Table 2. Lumbopelvic kinematics

\begin{tabular}{|c|c|c|c|c|}
\hline \multirow{2}{*}{ Lumbopelvic kinematics } & \multicolumn{2}{|c|}{ Mean $\pm^{\mathrm{c}} \mathrm{SD}\left({ }^{\circ}\right)$} & \multirow{2}{*}{$t$ value } & \multirow{2}{*}{$p$ value } \\
\hline & PHE & PHEIS & & \\
\hline Anterior tilting $\left(^{\circ}\right)$ & $8.9 \pm 1.2$ & $2.4 \pm 0.9$ & -7.82 & $<0.05$ \\
\hline Rotation $\left({ }^{\circ}\right)$ & $5.6 \pm 2.4$ & $3.1 \pm 1.8$ & -5.74 & $<0.05$ \\
\hline
\end{tabular}

PHE: Prone hip extension, PHEIS: Prone hip extension after iliopsoas stretching, SD: Standard deviation. 
change: $\left.4.67^{\circ}\right) .{ }^{21}$ However, Akbari and Mohammadi (2007) reported the flexibility of iliopsoas stretching carried out for 5 weeks, 3 times a week, session 2 min was not statistically different between group intervention ( $5 \mathrm{sec}$ vs. $15 \mathrm{sec}$ stretch). Both groups were increased length of the iliopsoas following iliopsoas stretching in healthy subjects. ${ }^{22}$ Although the intervention was 5 weeks, 3 times a week exercise program for hip flexibility, hip muscles strengthening exercise was not considered such as PHE. Considering that the results of the previous studies were meaningful, it is relatively clear whether iliopsoas stretching altered the amount of hip extension angle and might contributed to changing the muscle activation pattern as the our results demonstrated significantly greater GM activity in PHEIS for subjects with short iliopsoas. No previous studies have investigated this strategy to increase GM activity through lengthening of the iliopsoas before PHE in subjects with a short iliopsoas. Thus, it is not possible to compare the results from the previous studies.

There are several possible reasons for the increased GM activity shortly after iliopsoas stretching during PHE in subjects with iliopsoas shortness. First, the shortness of iliopsoas caused increased lumbar lordosis resulting in over activities of ES and MF. This back muscles dominance might cause hyper lordosis. In addition, short iliopsoas muscle increased pelvic anterior tilting during PHE because the insufficient length of iliopsoas pulling the origin in lumbar vertebra. Pelvic anterior tilting might provide lengthened position of GM as a concept of length-tension relationship. The iliopsoas stretching intervention used in this study may be effective to increase the length of iliopsoas as anterior tilting and rotation were decreased. These meant that the position of pelvic during PHE may be maintained in the same position as sufficient hip extension occurred. In this point, the distance between the origin and the insertion of GM might be optimal for facilitation of the GM during PHEIS. Second, iliopsoas shortness may cause the increased pulling force anteriorly in lesser trochanter on femur head during PHE. After iliopsoas stretching, the increased extensibility of iliopsoas might provide lesser pulling force compared to that before stretching. Although we did not measure pulling force (i.e., resistance while being stretched) and the changing distance of femur head while being stretched, the minimal of anterior pulling force can contributed to maintaining the constant axis of rotation of femur head in hip joint during PHEIS. This major contributors to minimized pulling force might increase the GM activity. Third, as angle of hip extension was increased after iliopsoas stretching assessed by the modified Thomas test, the more increased EMG activity of the GM contributed to decreased activity of the BF during PHE. Previous studies have reported that mobility and stability around the hip joint can be produced with synergistic muscles, such as the GM, the BF, and the ST. ${ }^{23,24}$ Because GM and hamstring muscles are the synergists of the hip extensors, ${ }^{1}$ these muscles were activated together and influence each other with different movements. In this study, because the range of hip extension during the exercise was increased after iliopsoas stretching, it can be explained that the decreased BF EMG amplitudes were seen in PHEIS and the decreased EMG amplitudes of this muscles may contribute to the increased GM EMG amplitude during the PHEIS exercise. Thus, this study suggests that iliopsoas stretching exercise is an effective method for facilitating GM activity during PHE.

This study has several limitations. First, we did not directly measure the distance of femoral anterior glide during applying pressure for iliopsoas stretch. Even though the exclusion criteria have the anterior gliding syndrome of femur head during PHE, the undetectable distance changing of femur position might be occurred with the pressure passively pulled by the hand. Further study should be investigated on the changing position of femur head with passively applied pressure. Second, this study recruited only male subjects with iliopsoas shortness, the findings cannot be generalized to all subject groups (i.e. impingement). Third, subjects performed the each exercise in the same order. The task order may affect the results. Fourth, this study was not investigated in long term effect. Further study should investigate the lasting effectiveness of iliopsoas stretching during PHE. Finally, we did not use ADIM to stabilize lumbopelvic region to figure out the effectiveness of only iliopsoas stretching. Further study is needed to combine the effect of iliopsoas stretching with ADIM intervention together.

\section{CONCLUSIONS}

This study compared PHE and PHEIS on EMG activities of ES, MF, GM and BF and lumbopelvic compensations and hip extension angle in subjects with a short iliopsoas. The findings of the study showed that the hip extension angle and GM activity were significantly greater and the lumbopelvic compensations and ES and BF were significantly lower in PHEIS compared with PHE in subjects with a short iliopsoas. Consequently, the results indicate that PHEIS could be more effective method than PHE for improving hip extension angle and GM activity in subjects with a short iliopsoas. Thus, iliopsoas stretch should be performed first, and then perform the PHE exercise. 


\section{Key Points}

Question Can iliopsoas stretching be effective to increase muscle activity of GM with minimized lumbopelvic compensations during PHE?

Findings The finding of this study reported that the muscle activity of GM and hip extension angle were more increased and those of ES and BF and lumbopelvic compensations were more decreased significantly after iliopsoas stretching compared to only PHE in subjects with short iliopsoas.

Meaning Iliopsoas stretching is an effective intervention in subjects with short iliopsoas during PHE.

\section{Article information}

Conflict of Interest Disclosures: None.

Funding/Support: None.

Acknowledgment: None.

\section{REFERENCES}

1. Sahrmann SA. Diagnosis and treatment of movement impairment syndromes. $1^{\text {st }}$ ed. Missouri: Mosby; 2002; Elsevier.

2. Neumann DA. Kinesiology of the musculoskeletal system: Foundation for rehabilitation $3^{\text {rd }}$ ed. 2017. Elsevier.

3. Kerrigan DC, Ehrenthal SR. A maladaptive gait abnormality in patients with lumbar spinal stenosis. J Back Musculoskelet Rehabil. 1996;7(1):53-57.

4. Janda V. Evaluation of muscular imbalance. In: Liebenson C, editor. Rehabilitation of the spine: a practitioner's manual. $1^{\text {st }}$ ed. Baltimore: Lippincott Williams \& Wilkins. 1996:97-112.

5. O'Sullivan K, Murray E, Sainsbury D. The effect of warmup, static stretching and dynamic stretching on hamstring flexibility in previously injured subjects. BMC Musculoskelet Disord. 2009;10:37.

6. Winters MV, Blake CG, Trost JS, et al. Passive versus active stretching of hip flexor muscles in subjects with limited hip extension: A randomized clinical trial. Phys Ther. 2004;84(9) :800-807.

7. Franz JR, Paylo KW, Dicharry J, Riley PO, Kerrigan DC. Changes in the coordination of hip and pelvis kinematics with mode of locomotion. Gait Posture. 2009;29(3) :494498.

8. Barton CJ, Kennedy A, Twycross-Lewis R, Woledge R, Malliaras P, Morrissey D. Gluteal muscle activation during the isometric phase of squatting exercises with and without a Swiss ball. Phys Ther Sport. 2014;15(1):39-46.

9. Kang SY, Jeon HS, Kwon OY, Cynn HS, Choi B. Activa- tion of the gluteus maximus and hamstring muscles during prone hip extension with knee flexion in three hip abduction positions. Man Ther. 2013,18(4) :303-307.

10. Willson JD, Binder-Macleod S, Davis IS. Lower extremity jumping mechanics of female athletes with and without patellofemoral pain before and after exertion. Am J Sports Med. 2008;36(8):1587-1596.

11. Jeon IC, Hwang UJ, Jung SH, Kwon OY. Comparison of gluteus maximus and hamstring electromyographic activity and lumbopelvic motion during three different prone hip extension exercises in healthy volunteers. Phys Ther Sport. 2016;22:35-40.

12. Lewis CL, Sahrmann SA. Muscle activation and movement patterns during prone hip extension exercise in women. J Athl Train. 2009;44(3):238-248.

13. Kendall FP, McCreary EK, Provance PG. Muscles testing and function, $5^{\text {th }} \mathrm{ed}$. Williams \& Wilkins, Baltimore, MD 2005.

14. Ayotte NW, Stetts DM, Keenan G, Greenway EH. Electromyographical analysis of selected lower extremity muscles during 5 unilateral weight-bearing exercises. J Orthop Sports Phys Ther. 2007;37:48-55.

15. Nyland J, Kuzemchek S, Parks M, Caboru D. Femoral anteversion influences vastus medialis and gluteus medius EMG amplitude: composite hip abductor EMG amplitude ratios during isometric combined hip abduction-external rotation. J Electromyogr Kinesiol 2004;14:255-261.

16. Sykes K, Wong YM. Electrical activity of vastus medialis oblique muscle in straight leg raise exercise with different angles of hip rotation. Physiotherapy 2003;89:423-430.

17. Cram JR, Kasman GS, Holtz J. Introduction to surface electromyography. Gaithersburg: Aspen; 1998.

18. Hermens HJ, Freriks B, Disselhorst-Klug C, Rau G. Development of recommendations for SEMG sensors and sensor placement procedures. J Electromyogr Kinesiol. 2000;10:361-374.

19. Kendall FP, McCreary EK, Provance PG. Muscles testing and function, $5^{\text {th }} \mathrm{ed}$. Williams \& Wilkins, Baltimore, MD 2005.

20. Malai S, Pichaiyongwongdee S, Sakulsriprasert P. Immediate effect of hold-relax stretching of iliopsoas muscle on transversus abdominis muscle activation in chronic nonspecific low back pain with lumbar hyperlordosis. $J$ Med Assoc Thai. 2015;98 Suppl 5:S6-11.

21. Shiwani. Comparison of muscle energy technique and post isometric relaxation on Iliopsoas tightness to improve flexibility in healthy young individuals. International Journal of Applied Research. 2017;3(3):16-21.

22. Akbari A, Mohammadi M. Effect of post isometric stretch duration on the hip joint extension in females with short 
iliopsoas muscle. Shahrekord University of Medical Sciences Journal. 2007;9(2):68-75.

23. Chance-Larsen K, Littlewood C, Garth A. Prone hip extension with lower abdominal hollowing improves the relative timing of gluteus maximus activation in relation to bi- ceps femoris. Manual Therapy. 2010;15:61-65.

24. Page P, Frank C, Lardner R. Assessment and treatment of muscles imbalance: the Janda approach. Champaign: $\mathrm{Hu}$ man Kinetics. 2009;78-80. 